\title{
Actinomycosis-An Unusual Case of an Uncommon Disease
}

\author{
Louis R. Petrone, MD, Jocelyn J. Sivalingam, MD, and Alexander R. Vaccaro, MD
}

Actinomycosis is a chronic granulomatous disease caused by any of several anaerobic organisms from the genus Actinomyces. Though previously thought to be a fungal infection, these organisms are actually caused by gram-positive, filamentous bacteria.

Human infection with actinomycotic organisms has been recognized since the end of the 19th century. ${ }^{1-3}$ In the preantibiotic era, such infections were severe and often fatal. Currently the true incidence of actinomycosis is difficult to define because many mild cases likely are eradicated by the common use of antibiotics. ${ }^{2,3}$ Improved dental hygiene has also contributed to the declining incidence of infection. ${ }^{2,3}$ It is generally agreed that actinomycosis has become an uncommon entity.

We describe a case of pelvic and sacral actinomycosis in a patient who had none of the traditional risk factors for this disease.

\section{Case Report}

A previously healthy 37 -year-old woman related a 2-week history of fevers as high as $104^{\circ} \mathrm{F}$ associated with chills, night sweats, nausea, diarrhea, anorexia, and malaise. She also reported she had left lower back and leg pain in the sciatic distribution for several months. There were no symptoms of upper respiratory tract infection, headache, dysuria, vaginal discharge, irregular menses, weight loss, tremors, or myalgias. She had had lower abdominal pain, for which she was evaluated by her gynecologist, who noted a fullness in her left adnexa and recommended a pelvic sonogram. The sonogram showed an ill-defined left adnexal mass, and magnetic resonance imaging (MRI) was recommended for further evaluation.

Her medical history was notable for a cholecystectomy in 1994. She was taking ibuprofen and

Submitted 24 March 1998.

From the Department of Family Medicine (LRP), the Department of Medicine (JJS), and the Department of Orthopedic Surgery (ARV), Jefferson Medical College, Philadelphia. Address reprint requests to Louis R. Petrone, MD, 2305 Fairmount Ave, Philadelphia, PA 19130. acetaminophen for the fevers and a triphasic oral contraceptive. She was allergic to codeine. She smoked one pack of cigarettes per day intermittently for 20 years and drank alcohol minimally. She was not currently sexually active; in the past she had consistently used condoms. She denied illicit drug use. There was no history of gynecologic problems, use of an intrauterine device (IUD), or bowel disease.

When examined, she was an anxious-appearing woman whose temperature was $99.5^{\circ} \mathrm{F}$, pulse rate 100 beats per minute, and blood pressure 92/68 $\mathrm{mmHg}$. Findings of examination of head, eyes, ears, nose, and throat were unremarkable; there was no cervical lymphadenopathy. Her chest was clear, and her heart sounds were regular and without murmurs. There were no abdominal masses, tenderness, or hepatosplenomegaly, and there was no edema of the extremities. Tenderness was present over the left sciatic notch. Strength and sensation in the lower extremities were symmetrical, as were reflexes; there was, however, a positive straight leg test (tension sign) indicating irritation of the sciatic nerve.

A complete blood cell count revealed a white blood cell count of $12,000 / \mu \mathrm{L}$ with 73 percent neutrophils and 6 percent band forms, a hemoglobin level of $10.7 \mathrm{~g} / \mathrm{dL}$, and platelet count of $507,000 / \mu \mathrm{L}$. Erythrocyte sedimentation rate was $103 \mathrm{~mm} / \mathrm{hr}$. Alkaline phosphatase was $184 \mathrm{U} / \mathrm{L}$, alanine aminotransferase $47 \mathrm{U} / \mathrm{L}$, and gammaglutamyltransferase $98 \mathrm{U} / \mathrm{L}$; other liver function tests were normal. Urine culture, viral hepatitis studies, antinuclear antibodies, tuberculin test (purified protein derivative [PPD]), and chest radiographs were all negative. The thyroid-stimulating hormone level was normal. Plain radiographs of the lumbosacral spine showed no evidence of abnormally advanced degenerative changes, lytic foci or bony destruction, or abnormal soft tissue swelling.

An MRI of the pelvis showed an infiltrative process involving the pyriformis, obturator, ilia- 
cus, and psoas muscles, the sciatic nerve and plexus, and the left side of the sacrum from S1 to S5 with diffuse bony infiltration. The mass was described as mostly solid with some small cystic or necrotic areas. There was tethering of bowel loops within the pelvis. Our diagnostic considerations included neoplasia, such as lymphoma, sarcoma, and malignant fibrous histiocytoma, and infection. Two biopsies guided by computed tomography (CT) showed only skeletal muscle and inflammatory cells. Cultures of the specimens were negative for routine organisms as well as acid-fast bacilli and fungus.

Open biopsy of the mass was then performed through an inguinal approach. At the time of surgery the muscle planes were distorted by a diffuse inflammatory reaction, making their mobilization and separation difficult. Minor fluctuant foci were palpated within the substance of the iliopsoas muscle complex, which when bluntly probed expressed approximately 10 to $15 \mathrm{cc}$ of purulent fluid. Gram-negative rods and gram-variable filamentous bacteria were isolated at 36 hours from cultures obtained in the operating room. The patient was treated empirically with imipenem-cilastatin (Primaxin) and began to improve clinically. The organisms were eventually identified as Fusobacterium nucleatum and Actinomyces israelii. Pathologic section revealed sulfur granules compatible with actinomycosis.

When the causative organisms were identified, the patient was given an 8-week course of highdose intravenous penicillin followed by an extended course of oral clindamycin. She has remained afebrile since starting the antibiotics and 2 years later feels well and has returned to work fulltime. Follow-up MRIs with gadolinium have shown substantial improvement in the inflammatory mass but persistent changes in the sacrum, the importance of which are unclear. Follow-up sedimentation rates have been normal.

\section{Discussion}

Actinomycosis is a bacterial infection that can affect virtually any site in the body. Disease in humans is most commonly caused by $A$ israelii. ${ }^{1}$ Other species capable of causing human infection, although less frequently, are Actinomyces odontolyticus and Actinomyces viscosus. ${ }^{2}$ These organisms have been isolated from mucous membranes of the mouth, bronchi, gastrointestinal tract, and female genital tract. They are not considered particularly virulent pathogens, but rather opportunistic ones, because infection occurs usually only after disruption of the mucous membranes. The disease spreads by direct extension into surrounding tissues without regard for tissue planes through the formation of sinus tracts that can lead directly to the skin. The typical sulfur granules can drain from these tracts. Microscopically these infections are lobulated microcolonies of the organism.

Actinomycosis is traditionally divided into three forms: cervicofacial, thoracic, and abdominogenital. ${ }^{1,4}$ The most frequent site of human infection is the cervicofacial area, accounting for about 40 to 50 percent of cases. ${ }^{1-4}$ Poor dental hygiene or trauma to the mucous membranes of the oral cavity is usually responsible for cervicofacial actinomycosis. ${ }^{5}$ Approximately 15 percent of actinomycosis occurs in the thorax. ${ }^{1,4}$ Infection here can be caused by direct extension from an existing infection in the head and neck region or as the result of aspiration of the organism from the throat. Twenty percent of actinomycotic infections occur in the abdomen and pelvis. ${ }^{2,4}$

Abdominal disease usually results from clinical or subclinical disruption of bowel mucosa. It often occurs as a firm mass that appears fixed to the surrounding tissue and can be mistaken for tumor. ${ }^{2,6-8}$ An intra-abdominal event, such as appendicitis or perforated viscus, could give rise to pelvic actinomycosis. ${ }^{9,10}$ In the early 1970 s a rise in the incidence of pelvic infection was attributed to intrauterine contraceptive devices (IUDs). ${ }^{11}$ When pelvic actinomycosis occurs, it usually causes endometritis, salpingo-oophoritis, or tubo-ovarian abscess, ${ }^{12}$ and a mass in the adnexa might be palpable, suggesting a pelvic malignancy.,8 Ultimately, extension to the abdominal wall or deep pelvic structures can occur.

Actinomycosis can also occur in bone, usually as the result of direct extension from a nearby infection. ${ }^{13}$ In the preantibiotic era, the vertebrae were frequently infected bones, predictive of a mortality rate as high as 76 percent. ${ }^{14}$ Recently, however, given the relative frequency of orofacial actinomycosis and the decrease in the occurrence of widespread disease, the facial bones, particularly the mandible, are the most frequent sites of osseous infection. ${ }^{14}$ Current studies have found that the incidence of vertebral infection is quite low. In one study of 181 cases, lung abscesses ex- 
tended to vertebral bone in only two cases. ${ }^{15}$ Other smaller studies found no cases of vertebral involvement. ${ }^{1,4,5}$

Hematogenous spread to other organs, such as the brain, liver, and kidney, can occur but is quite uncommon. Central nervous system involvement occurs in approximately 3 percent of cases, usually as a result of dissemination from a primary lung focus $^{3}$ or extension of cervicofacial disease.

Actinomycosis has been noted to be a polymicrobial infection ${ }^{2-4}$; it is often found along with another organism, such as Fusobacterium in our patient. Other bacteria that are isolated with $A c$ tinomyces include anaerobic streptococci, certain Haemophilus species, and various gram-negative bacilli. ${ }^{1}$ It is possible that these organisms act as cofactors that allow the Actinomyces organisms to propagate.

Diagnosis of actinomycosis can be difficult because of the insidious nature of the infection. Many times the diagnosis is missed preoperatively, even after fine-needle aspiration. ${ }^{16}$ The finding of sulfur granules from any site other than the tonsils is considered pathognomonic. ${ }^{1-3}$ CT- or sonographic-guided biopsy can be used to obtain material for diagnosis. Occasionally, as with our patient, surgery could be required.

Once the organism is identified, treatment is relatively straightforward. Most strains of Actinomyces are sensitive in vitro to a variety of agents, such as tetracycline, erythromycin, cephalosporins, and clindamycin. ${ }^{16}$ Ciprofloxacin and imipenem-cilastatin have also been used. ${ }^{17,18}$ It is generally recommended, however, to treat with penicillin, to which the organisms are usually sensitive, because of its low cost, its high tolerability, and the extent of clinical experience. ${ }^{1,5}$ Long-term therapy, lasting from 8 weeks to several years, is usually recommended. ${ }^{1,9}$ Generally, the disease is treated until there is evidence of complete resolution. Surgery is occasionally needed to drain abscesses. ${ }^{5,9}$ but because actinomycotic infection does not follow tissue planes, surgery can be complicated and, if possible, should be delayed at least until after a course of antibiotics has been given.

The prognosis of actinomycosis in the current era of antibiotics is usually very good. If diagnosis is delayed, however, extensive local involvement can develop. Furthermore, if the disease disseminates, especially to the brain, death can uccur. ${ }^{1}$

The evolution of disease in our patient is inter- esting for several reasons. First, she had no identifiable source of pelvic infection; that is, she had never used an IUD, nor did she have a history of abdominopelvic trauma or disease. She had had an uncomplicated cholecystectomy approximately 1 year before onset of her symptoms, and although actinomycosis of the gallbladder has been reported,${ }^{19}$ it is unlikely that her gallbladder was the source of infection because there was no evidence of direct extension from the right upper quadrant. She had had no other gastrointestinal or pelvic procedures before her symptom onset. Findings from a barium enema and a follow-up gynecologic examination after the diagnosis was made were completely normal. Hematogenous seeding is unlikely because other sites of disease would be expected throughout the body. Furthermore, there was no history of recent dental work, and findings from a dental examination after the diagnosis was made were completely normal. We postulate that a microperforation could have occurred in her bowel or genital tract that allowed access of the organisms to the deep pelvis. Another possibility is that with routine sexual intercourse the organisms could have been expressed through the fallopian tubes into the pelvis.

Another unusual aspect of this case is that our patient had extensive sacral involvement of infection, which to our knowledge has not been reported.

Finally, neurologic complications of actinomycosis are usually the result of direct pressure of the inflammatory mass on a nerve or on the spinal cord. Cases of epidural involvement by Actinomyces organisms resulting in paraparesis have been reported. ${ }^{20,21}$ Our patient had symptoms of sciatic nerve compression, a result of compression by the infectious mass. Sciatica has not been reported in several series of cases of actinomycosis. $4,5,15$

\section{Summary}

Actinomycosis is an uncommon disease caused by organisms of the Actinomyces genus. These organisms are commonly found in the mucous membranes but do not cause infection unless there is disruption of the membranes, as occurs, for example, during dental trauma or abdominal surgery. Use of an IUD is also a risk factor for pelvic actinomycosis. The disease is usually insidious and is often mistaken for other conditions. Treatment of the infection, once diagnosed, is a regimen of 
long-term antibiotics such as penicillin, clindamycin, and others. Our patient had pelvic and sacral actinomycosis without any of the traditional risk factors for infection.

\section{References}

1. Bennhoff DF. Actinomycosis: diagnostic and therapeutic considerations and a review of 32 cases. Laryngoscope 1984;94:1198-217.

2. Russo TA. Agents of actinomycosis. In: Mandell GL, Bennett JE, Dolin R, editors. Mandel, Douglas and Bennett's principles and practice of infectious disease. 4th edition. New York: Churchill Livingstone, 1995.

3. Actinomycosis. In Rippon JW. Medical mycology: the pathogenetic fungi and the pathogenic actinomycetes. 3rd edition. Philadelphia: WB Saunders, 1988.

4. Weese WC, Smith IM. A study of 57 cases of actinomycosis over a 36-year period. A diagnostic 'failure' with good prognosis after treatment. Arch Intern Med 1975;135:1562-8.

5. Eastridge CE, Prather JR, Hughes FA Jr, Young JM, McCaughan JJ Jr. Actinomycosis: a 24 year experience. South Med J 1972;65:839-43.

6. Fowler RC, Simpkins KC. Abdominal actinomycosis: a report of three cases. Clin Radiol 1983;34:301-7.

7. Perlow JH, Wigton T, Yordan EL, Graham J, Wool N, Wilbanks GD. Disseminated pelvic actinomycosis presenting as metastatic carcinoma: association with the Progestasert intrauterine device. Rev Infect Dis 1991;13:1115-9.

8. Hinnie J, Jacques BC, Bell E, Hansell DT, Milroy R. Actinomycosis presenting as carcinoma. Postgrad Med J 1995;71:749-50.

9. Wohlgemuth SD, Gaddy MC. Surgical implications of actinomycosis. South Med J 1986;79:1574-8.

10. Ellis LR, Kenny GM, Nellans RE. Urogenital aspects of actinomycosis. J Urol 1979;122:132-3.

11. Henderson SR. Pelvic actinomycosis associate with an intrauterine device. Obstet Gynecol 1973;41: 726-32.

12. Valicenti JF Jr, Pappas AA, Graber CD, Williamson HO, Willis NF. Detection and prevalence of IUDassociated Actinomyces colonization and related morbidity. A prospective study of 69,925 cervical smears. JAMA 1982;247:1149-52.

13. Young WB. Actinomycosis with involvement of the vertebral column: case report and review of the literature. Clin Radiol 1960;11:175-82.

14. Lewis RP, Sutter VL, Finegold SM. Bone infections involving anaerobic bacteria. Medicine-Baltimore 1978;57:279-305.

15. Brown JR. Human actinomycosis: A study of 181 subjects. Hum Pathol 1973;4:319-30.

16. Fiorino AS. Intrauterine contraceptive device-associated actinomycotic abscess and Actinomyces detection on cervical smear. Obstet Gynecol 1996;87: 142-9.

17. Macfarlane DJ, Tucker LG, Kemp RJ. Treatment of recalcitrant actinomycosis with ciprofloxacin. J Infect 1993;27:177-80.

18. Yew WW, Wong PC, Wong CF, Chau CH. Use of imipenem in the treatment of thoracic actinomycosis. Clin Infect Dis 1994;19:983-4.

19. Smithers BM, Wall DR, Weedon D. Actinomycosis of the gallbladder. Aust N Z J Surg 1983;53:587-8.

20. Muller PG. Actinomycosis as a cause of spinal cord compression: a case report and review. Paraplegia 1989;27:390-3.

21. Kannangara DW, Tanaka T, Thadepalli H. Spinal epidural abscess due to Actinomyces israelii. Neurology 1981;31:202-3. 